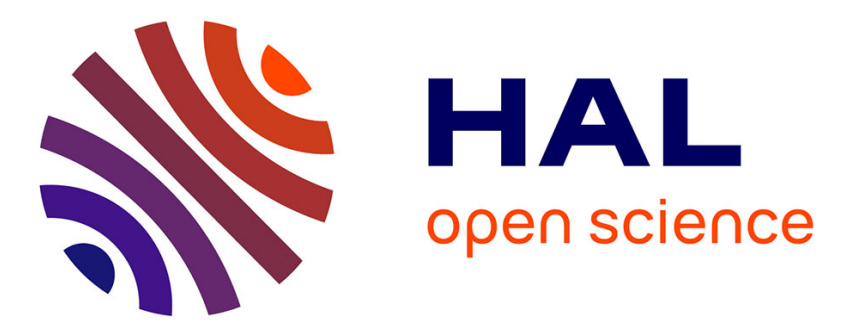

\title{
Comparison of different coupling schemes between counterions and charged nanoparticles in multiparticle collision dynamics
}

Vincent Dahirel, Xudong Zhao, Marie Jardat

\section{- To cite this version:}

Vincent Dahirel, Xudong Zhao, Marie Jardat. Comparison of different coupling schemes between counterions and charged nanoparticles in multiparticle collision dynamics. Physical Review E , 2016, 94, pp.023317. 10.1103/PhysRevE.94.023317 . hal-01472394

\section{HAL Id: hal-01472394 \\ https: / hal.sorbonne-universite.fr/hal-01472394}

Submitted on 31 Jan 2020

HAL is a multi-disciplinary open access archive for the deposit and dissemination of scientific research documents, whether they are published or not. The documents may come from teaching and research institutions in France or abroad, or from public or private research centers.
L'archive ouverte pluridisciplinaire HAL, est destinée au dépôt et à la diffusion de documents scientifiques de niveau recherche, publiés ou non, émanant des établissements d'enseignement et de recherche français ou étrangers, des laboratoires publics ou privés. 


\title{
Comparison of different coupling schemes between counterions and charged nanoparticles in multiparticle collision dynamics
}

\author{
Vincent Dahirel, Xudong Zhao, Marie Jardat \\ Sorbonne Universités, UPMC Univ Paris 06, CNRS, UMR 8234, PHENIX, F-75005 Paris, France
}

(Dated: August 9, 2016)

\begin{abstract}
We applied the multiparticle collision dynamics (MPC) simulation technique to highly asymmetric electrolytes in solution, i.e. charged nanoparticles and their counterions in a solvent. These systems belong to a domain of solute size which ranges between the electrolyte and the colloidal domains, where most analytical theories are expected to fail, and efficient simulation techniques are still missing. MPC is a mesoscopic simulation method which mimics hydrodynamics properties of a fluid, includes thermal fluctuations and can be coupled to a molecular dynamics of solutes. We took advantage of the size asymmetry between nanoparticles and counterions to treat the coupling between solutes and the solvent bath within the MPC method. Counterions were coupled to the solvent bath during the collision step, and nanoparticles either through a direct interaction force or with stochastic rotation rules which mimic stick boundary conditions. Moreover, we adapted the simulation procedure to address the issue of the strong electrostatic interactions between solutes of opposite charges. We show that the short-ranged repulsion between counterions and nanoparticles can be modeled by stochastic reflection rules. This new simulation scheme is very efficient from a computational point of view. We have also computed the transport coefficients for various densities. The diffusion of counterions was found in one case to increase slightly with the volume fraction of nanoparticles. The deviation of the electric conductivity from the ideal behavior (solutes at infinite dilution without any direct interactions) is found to be strong.
\end{abstract}

\section{INTRODUCTION}

Charged nanoparticles are ubiquitous in lots of fluids of biological, ecological or industrial interest. Some of the properties of these fluids are governed by the transport properties of the nanoparticles. Moreover, the characteristics of the nanoparticles themselves like their size or their charge is often deduced from an interpretation of their transport coefficients. For instance, widely used analytical tools in labs are zetametry ${ }^{1,2}$, which gives the zeta potential of nanoparticles from their electrical mobility, or dynamic light scattering ${ }^{3}$, which allows to measure a diffusion coefficient from which the approximate radius of the nanoparticle can be deduced. Even in dark dense media, there are ways to characterize the size of the particles by measuring other transport coefficients, such as the electroacoustic potential ${ }^{4,5}$.

Despite the interest of transport coefficients in these fluids, their theoretical prediction still remains a challenge, even in the case where interactions within the system are well understood. For example, a solution of charged globular proteins, with a radius of the protein of about one nanometer, neutralized by its counterions, can be considered as a colloidal system. But, as colloidal particles are here very small, usual colloidal theories are expected to fail ${ }^{6}$. Alternatively, this system can be handled as an highly asymmetric electrolyte, but with such an asymmetry in charge and in size between ions of opposite charge, this system is much more difficult to describe than simple electrolytes such as $\mathrm{NaCl}$ or $\mathrm{CaCl}_{2}$ in water. Usual electrolyte theories are therefore also expected to fail ${ }^{7}$.

For these kinds of systems where typical colloidal or electrolyte theories should be used with much caution, investigations through numerical simulations are important. Molecular Dynamics (MD) at the atomistic level is a possibility, but computing for instance the electrical conductivity of a protein solution using such a detailed modelling requires a very substantial use of computational capabilities. There are two main reasons for that. First, the electrical conductivity is a collective transport coefficient, which can be defined from the integral over time of the autocorrelation function of the total electric current within a given volume of the liquid, such as a simulation box ${ }^{8,9}$. This quantity is much more difficult to compute with a good precision than individual transport coefficients, such as the self-diffusion coefficient. Indeed, the later can be deduced from the integral over time of the autocorrelation function of individual velocities, which can be averaged out over the number of solute particles. Second, the time scale at which the integral of autocorrelation functions converges is of the order of a few nanoseconds for such systems: Getting precise values of these long time properties requires very long computed trajectories. Therefore, using alternative coarse-grained simulations is still necessary, despite the progress of computational facilities. At least the description of the solvent must be simplified, with all the care required if one wants to reduce the number of degrees of freedom explicitly described in a system.

Dynamic properties of charged species in solution are influenced by thermal fluctuations, by direct electrostatic interactions and by hydrodynamic interactions mediated by the solvent. The numerical simulation of fluids containing nanoparticles thus requires the correct modeling of all these direct and indirect interactions mediated by the solvent. When the case of charged nanoparticles is investigated, the presence of a possibly large amount of 
small counter- and co-ions makes the simulation harder. For some methodologies, this difficulty lies not only in their abundance but also in their small size (compared to nanoparticles). In particular, describing solutes of different sizes requires to correctly describe hydrodynamics at different length scales. Hydrodynamic interactions are long-range, compared to the size of the solute particles. Therefore, in asymmetric systems, the size of the simulation box must be much larger than that of the biggest solute particle in order to resolve hydrodynamic interactions far from these particles. Besides this constraint, the fluid velocity field at the surface of the solute particles must also be carefully resolved, and therefore the description of hydrodynamics around smaller ions have to be carefully handled.

Moreover, another difficulty emerges due to the intensity of electrostatic interactions, for instance in situations where electrostatic condensation is expected to happen ${ }^{10}$. In most coarse-grained simulation techniques, dealing with strong interactions is avoided if possible, especially because one objective of coarse-grained simulations is to increase the time step compared to atomistic ones ${ }^{11}$. Coarse-grained simulation algorithms like Dissipative Particle Dynamics ${ }^{12}$ or Brownian dynamics ${ }^{13-16}$ often include a stochastic component, which is a consequence of the implicit description of the solvent. Therefore, electrostatically condensed ions are more likely to explore unstable regions of phase space with stochastic algorithms than with MD: The random component of their dynamics can lead them there. This problem disappears when the time step decreases, but otherwise it can lead to unphysical trajectories. This problem is particularly important when the stochastic component directly impacts the positions of the solutes, like in Brownian Dynam$\operatorname{ics}^{17-19}$, but it is less consequential when the stochastic component impacts the velocities, like in multiparticle collision dynamics, the mesoscopic methodology chosen in this study ${ }^{20-22}$.

One possibility to go beyond the simplification of the sole solvent and to overcome the problem due to the strong interactions between the nanoparticles and their counterions is to use an effective interaction potential between nanoparticles ${ }^{6,23,24}$ and to get rid of the explicit description of small ions. Nevertheless, it is well known that small ions play in some cases an important dynamical role. One can define effective dynamical quantities, such as an effective diffusion coefficient ${ }^{25}$, accounting for the effect of the removed degrees of freedom, but still for some applications the explicit description of counterions is mandatory. For instance, an effective diffusion coefficient would not account properly for the effects of counterions in non-equilibrium simulations, e.g. under the presence of an electric field ${ }^{26,27}$. Moreover, the computation of some collective transport coefficients, such as the electrical conductivity, or even the mutual diffusion coefficient which is measured by Dynamic Light Scattering, requires an explicit description of the counterions ${ }^{28,29}$.

In this context, our purpose in this work is to adapt the multiparticle collision dynamics simulation (MPC) method to systems which contain charged nanoparticles and their counterions. MPC is an efficient mesoscopic simulation method to describe the dynamic properties of fluids in various regimes ${ }^{20-22}$. In this algorithm, the fluid is represented by point like particles that evolve in two steps. Ballistic displacements of solvent particles are followed by collision steps where solvent particles interact through a momentum exchange. As this algorithm conserves momentum and energy, it generates the correct Navier-Stokes hydrodynamics. This bath of solvent particles can be coupled to a molecular dynamics simulation of the trajectories of solute particles ${ }^{21,22}$.

Several variants of the coupling scheme between solutes and solvent exist. This part of the methodology is particularly important because, as we proceed to show, this is where the asymmetry between the nanoparticle and its counterions can be exploited to decrease the computation time. The simplest way to couple solutes with the solvent is to assume that solutes interact with the solvent only during the collision step, exchanging momentum with solvent particles ${ }^{20,22}$. In this case, no excluded volume exists for the solvent. We refer to this scheme as CC (Collisional Coupling) in what follows. The excluded volume between solutes and solvent can be taken into account via explicit repulsive forces, deriving for example from a Weeks-Chandler-Andersen interaction potential $^{30}$. We refer to this scheme as CFC (Central Force Coupling) in what follows. The CFC coupling leads to a slip boundary condition of the solvent at the surface of the solute ${ }^{21}$. Stick boundary conditions between solutes and solvent can be mimicked thanks to stochastic reflections of the solvent at the surface of the solute $^{31-34}$. We refer to this scheme as SRR (Stochastic Reflections Rules) in what follows. We have shown recently that, for neutral solutes and symmetric electrolytes, the CFC MPC scheme compares very well with Brownian Dynamics simulations accounting for hydrodynamic interactions through the Rotne Prager hydrodynamic tensor ${ }^{35,36}$. The later methodology is known to predict well the effect of the volume fraction of charged solutes on the transport coefficients ${ }^{16,37,38}$. The CC scheme is less effective in taking into account the effect of the size of solutes and therefore the influence of volume fraction on transport. However, the CC algorithm is more efficient from a computational point of view, and is a good compromise for systems for which a precise description of the hydrodynamic size is not mandatory. This is the case for small ions in water: The size difference between ions and solvent molecules is small, and the hydrodynamic size of the ion is not well defined from structural data, such as the cristallographic size or from the size of the fully hydrated ion. The description of continuous hydrodynamics around an ion is deduced from an empirical top-down strategy $^{39}$. The hydrodynamic boundaries of small ions are not well defined microscopically, and the use of the collisional coupling seems justified in this case. On the other hand, this is not the case for colloidal particles with 
clear hydrodynamic boundaries ${ }^{40}$.

We propose here an efficient scheme based on MPC simulations to compute the dynamic properties of charged nanoparticles in solution in the presence of small counterions. We suggest to use the CC coupling scheme between the solvent and the small counterions, and either CFC or SRR coupling schemes between nanoparticles and the solvent bath. Moreover, to avoid the time consuming computation of short-range interactions between counterions and nanoparticles, and to increase the stability of the algorithm when the MD time step is increased, we propose to use SRR conditions for counterions in the vicinity of nanoparticles, using the same procedure as for the solvent. It allows us to bypass the problem of the exploration of the potential energy surface for highly attracted charged solutes. Indeed, within this algorithm, the short range repulsive interaction is replaced by an exclusion scheme resulting in effective hard core interactions. The discretization of the trajectory using finite time steps is no longer a source of instability. The However, it is important to check whether this approximate scheme, which affects the dynamics of the counterions at the surface of the nanoparticle, leads to transport coefficients that are quantitatively similar to the reference scheme, where explicit interactions between counterions and nanoparticles would be used.

In what follows, we study three different systems which differ by the intensity of the electrostatic attraction between nanoparticles and counterions, with three different variants of the MPC algorithm. In every case, small ions are coupled to the solvent bath through a CC scheme. We show that the dynamic properties computed with SRR for small ions in the vicinity of nanoparticles are very close to those obtained with explicit repulsive forces, even for strong electrostatic attractions between them. Moreover, this procedure demands a computation time about twenty times shorter than the usual one for the systems investigated here. We also investigate the dynamic properties of nanoparticles and small ions with slip boundary conditions of the solvent on nanoparticles (CFC scheme), and we show that the nanoparticle/solvent boundary condition also influences the dynamics of counterions.

Section II of this paper details the simulation methods, section III describes the systems under study and contains the parameters of simulations. In section IV we compare the results obtained with the different schemes, and section $\mathrm{V}$ presents the application of the new simulation scheme to the study of the influence of the volume fraction on dynamic properties of solutes.

\section{SIMULATION METHODS}

\section{A. MPC algorithm: Pure fluid}

The MPC algorithm for a pure fluid is already described elsewhere $\mathrm{e}^{20-22}$, and we only recall here the main lines. The fluid is described by point-like particles (the
MPC fluid), whose positions and velocities evolve in two steps. First, in the streaming step, the particles evolve like in molecular dynamics, through the integration of Newton's equations of motion. There is no force between the fluid particles, thus the only forces acting on the MPC fluid are due to external fields, solutes or walls. Therefore, the computation time associated with this step scales as the number $N$ of fluid particles. This is the major advantage of the methodology. The exchange of momentum between the fluid particles occurs in a second step, the collision step. The simulation box is divided into cubic collision cells. For each collision cell, a randomly oriented axis is chosen. The velocity of each fluid particle relative to the velocity of the center of mass of the cell is rotated by an angle $\alpha$ around this axis:

$$
\mathbf{v}_{i}\left(t+\delta t_{c}\right)=\mathbf{v}_{c . o . m}^{\text {cell }}(t)+\mathcal{R}_{\alpha}\left[\mathbf{v}_{i}(t)-\mathbf{v}_{c . o . m}^{\text {cell }}(t)\right]
$$

where $\mathbf{v}_{i}$ is the velocity of particle $i, \mathcal{R}_{\alpha}$ is the rotation matrix, $\mathbf{v}_{\text {c.o.m }}^{\text {cell }}$ the velocity of the center of mass of the cell, and $\delta t_{c}$ is the time between two collision steps. This second step locally mimics interactions between the fluid particles. The hydrodynamic properties of the fluid depend on the choice of the angle $\alpha$, of the mean number of fluid particles per unit cell $\gamma$, and on the duration $\lambda$ of the streaming step, i.e the period between two collisions.

\section{B. Coupling the fluid and the solutes}

There are several ways to couple the MPC fluid to explicit particles embedded in this fluid. In the following, we refer to the MPC fluid as the solvent, and to the embedded particles as the solutes.

The simplest coupling scheme is to couple the solvent and solutes particles during the collision step. In this paper, we will refer to this scheme as Collisional Coupling (CC). See a description in $e g^{22,41}$. Within this scheme, the solvent particles can enter the solute particles, and therefore the effect of the size of the solute particles on the long range hydrodynamics is not quantitatively reproduced. Nevertheless, it has been used for instance for polymers ${ }^{42-44}$, and it was shown to be successful in describing the effect of hydrodynamics on the polymer dynamics.

A second possibility is to add a central repulsive force between the solvent and the solute particles. In this case, the coupling occurs during the streaming step. In this paper, we will refer to this scheme as Central Force Coupling (CFC). In this case, the solvent is excluded from the solute, and it slips at the surface of the solute. The solute has then an explicit hydrodynamic size, which is however different from the typical hydrodynamic radius in colloidal dynamics, due to the presence of a non-hydrodynamic source of friction, the Enskog friction (for more details, see reference ${ }^{21}$ ). Beyond that difficulty, this scheme requires quite small integration time steps to 
resolve the solvent dynamics around the solute, therefore it increases substantially the computational cost. In the present paper, when this coupling mode is chosen, we use a Weeks-Chandler-Andersen (WCA) interaction potential between the solvent and solute:

$U_{\text {wca }}(r)= \begin{cases}4 \epsilon_{\mathrm{wca}}\left[\left(\frac{\sigma}{r}\right)^{12}-\left(\frac{\sigma}{r}\right)^{6}\right]+\epsilon_{\mathrm{wca}} & \text { if } r<2^{1 / 6} \sigma \\ 0, & \text { otherwise }\end{cases}$

where $\epsilon_{\mathrm{wca}}$ controls the intensity of the repulsion, $\sigma$ is a size parameter, which is larger than the hydrodynamic size of the solute to avoid spurious depletion effects ${ }^{21}$. As the interaction potential is rather steep, the streaming step must be divided into smaller MD time steps, denoted by $\Delta t$, in order to ensure the stability of the algorithm.

A third family of methods intends to reproduce no-slip boundary conditions at the surface of the solute (or of a wall). This condition is a better representation of solutes as colloidal particles, since attractive short-ranged interactions with the solvent are expected to stick solvent molecules at the surface of the particles ${ }^{31,32}$. In the present study, we used the Stochastic Reflection Rules algorithm (SRR). The SRR for solvent particles around nanoparticles was first proposed by Inoue et $a l^{45}$. Within this scheme, when a solvent particle enters a solute particle, the time and position of the impact is computed, the solvent particle is restored to this impact point and is given a random velocity obtained through a half-plane Maxwell-Boltzmann distribution. For the remainder of the step, the solvent particle streams from the point of contact using this random velocity. The following modification of the algorithm has been proposed in ref. ${ }^{31}$. Instead of computing the exact impact time when a solvent particle enters the solute, the dynamics is reversed so that both solvent and solutes go half a step back. Then, the position of the solvent particle is replaced by the closest point at the surface of the solute. After that, a random velocity is generated, which is used to propagate the solvent particles for the remaining half time step.

We use here the implementation of the algorithm proposed by Padding et al and described in ref. ${ }^{31}$. The assigned position of the solvent particle at the surface of the solute particle is denoted by $r^{*}$. In order to implement the momentum exchange between the solvent particle $i$ and the solute or nanoparticle NP, the solvent particle is assigned random normal and tangential velocities $v_{n}^{*}$ and $v_{t}^{*}$ respectively (relative to the velocity of the nanoparticle). The probability distribution for the scattered velocity is given by

$$
\begin{gathered}
P\left(v_{n}^{*}\right)=m \beta v_{n}^{*} \exp \left(-m \beta v_{n}^{* 2} / 2\right) \\
P\left(v_{t}^{*}\right)=\sqrt{m \beta /(2 \pi)} \exp \left(-m \beta v_{t}^{* 2} / 2\right)
\end{gathered}
$$

where $\beta=\left(k_{B} T\right)^{-1}$ with $k_{B}$ the Boltzmann constant, $T$ the temperature, $m$ the mass of a solvent particle.
After the impact of a solvent particle $i$ with a solute particle $j$, the velocity of solvent particle $i$, denoted by $\mathbf{v}_{i}$, becomes:

$\mathbf{v}_{i}(t+\Delta t)=\mathbf{V}_{j}^{\mathrm{NP}}(t)+\boldsymbol{\Omega}_{j}^{\mathrm{NP}} \times\left[\mathbf{r}^{*}-\mathbf{R}_{j}(t)\right]+v_{n}^{*} \hat{\mathbf{e}}_{n}+v_{t}^{*} \hat{\mathbf{e}}_{t}$

where $\hat{\mathbf{e}}_{n}$ and $\hat{\mathbf{e}}_{t}$ are the unit vector in normal and tangential directions, $\mathbf{R}_{j}$ is the position vector of the solute particle $j, \boldsymbol{\Omega}_{j}^{\mathrm{NP}}$ is the angular velocity of the solute particle $j, \mathbf{V}_{\mathbf{j}}{ }^{\mathrm{NP}}$ is the velocity of the solute particle $j$. The final velocity of the nanoparticle particle $j$ encountered by a solvent particle $i$ becomes:

$$
\mathbf{V}_{j}^{\mathrm{NP}}(t+\Delta t)=\mathbf{V}_{j}^{\mathrm{NP}}(t)+\frac{m}{M}\left[\mathbf{v}_{i}(t)-\mathbf{v}_{i}(t+\Delta t)\right]
$$

where $M$ is the mass of the nanoparticle $j$. The angular velocity of the nanoparticle is modified as well:

$$
\begin{aligned}
\boldsymbol{\Omega}_{j}^{\mathrm{NP}}(t+\Delta t)= & \mathbf{\Omega}_{j}^{\mathrm{NP}}(t)+ \\
& \frac{m}{I}\left[\mathbf{r}^{*}-\mathbf{R}_{j}(t)\right] \times\left[\mathbf{v}_{i}(t)-\mathbf{v}_{i}(t+\Delta t)\right]
\end{aligned}
$$

with the moment of inertia of the nanoparticle $I=$ $M d^{2} / 10$ with $d$ the diameter of the nanoparticle.

Within this methodology, the question of the discretization of the equation of motion in the presence of a steep interaction potential vanishes. It is not necessary to divide the streaming step into smaller MD steps, except if the density of solutes is important. In this case, multiple reflections on the solute surface are expected to become more likely, and a good way to avoid them is to divide the streaming step.

\section{Stochastic Reflection Rules for counterions}

In the case of charged colloidal systems, the issue of the discretization of the equations of motion for counterions close to the surface of the nanoparticle is even more problematic than in the case of solvent particles. As counterions are attracted by the nanoparticle, they are concentrated close to the surface. Using a short-ranged repulsive interaction potential to mimic the excluded volume of the nanoparticle renders thus the description of the dynamics of electrostatically condensed ions challenging.

In order to address this issue, we propose to apply the Stochastic Reflection Rules mentioned above to counterions in the vicinity of nanoparticles. The same algorithm is used for counterions and solvent particles when they encounter a nanoparticle. The translational and angular velocities are modified as well, following eqns. 5-7. The finite size of the counterions in the real solution is taken into account by replacing the radius of the nanoparticle in eqns.5-7 by the sum of the nanoparticle radius and that of the counterions. 
In the framework of this new scheme, the only forces to be explicitly computed in the system are actually Coulomb forces. In particular, short-range repulsive forces between counterions which would account for their finite size are not taken into account. This does not lead to unphysical situations because Coulomb interactions between counterions are repulsive. It would be more tricky to handle the case of added salt, with Coulomb attraction between ions of opposite size. We are working on this issue which will be dealt with in a forthcoming article. Finally, this method is computationally lighter than the usual one where all interactions between charged species are taken into account. Moreover, larger time steps can be used.

\section{Summary of the different coupling schemes used}

In the current study, our main effort is focused on the comparison of three algorithms adapted to the simulation of the dynamic properties of charged nanoparticles and their counterions in suspension in the framework of MPC. In all cases, nanoparticles interact with each other through Coulomb interactions and a WCA potential with parameters $\epsilon_{N P}$ and $\sigma_{N P}$. Nanoparticles and their counterions interact through Coulomb interactions. Direct interactions between counterions consist in Coulomb interactions and in short-ranged repulsions with parameters $\epsilon_{c}$ and $\sigma_{c}$ in two cases over three. In every case, long-ranged Coulomb interactions are computed using the usual Ewald summation technique ${ }^{46}$.

In all three cases, counterions are coupled to the solvent within the collision steps, i.e we use the $\mathrm{CC}$ coupling scheme for these small solutes. The nanoparticles are coupled to the solvent bath either through WCA interactions (CFC algorithm) with parameters $\epsilon_{N P / f}$ and $\sigma_{N P / f}$, or by using the Stochastic Reflection Rules mimicking no-slip boundary conditions (SRR algorithm). With SRR, the minimal distance of approach between the solvent and the nanoparticle is called $\sigma_{N P / f}$.

When nanoparticles are treated within the CFC algorithm, counterions also interact with nanoparticles through a central WCA potential, with parameters $\epsilon_{N P / c}$ and $\sigma_{N P / c}$, and they interact with each other through a WCA potential with parameters $\epsilon_{c}$ and $\sigma_{c}$. We therefore call this method F-CFC, for Full Central Force Coupling.

When SRR conditions are used for the solvent in the vicinity of nanoparticles, interactions between counterions and nanoparticles are treated in two different ways. In the first case, counterions interact with nanoparticles through a WCA potential with parameters $\epsilon_{N P / c}$ and $\sigma_{N P / c}$ in addition to Coulomb interactions. Counterions interact with each other through Coulomb interactions and short-ranged WCA repulsions with parameters $\epsilon_{c}$ and $\sigma_{c}$. This method is called SRR-CFC, as it combines $\mathrm{SRR}$ for solvent/nanoparticle and $\mathrm{CFC}$ for counterions/nanoparticle), and differs from the F-CFC variant in the treatment of solvent/nanoparticle inter-
TABLE I: Different coupling schemes

\begin{tabular}{|l|l|l|}
\hline $\begin{array}{l}\text { Acronym of } \\
\text { the method }\end{array}$ & $\begin{array}{l}\text { solvent/nanoparticle } \\
\text { interactions }\end{array}$ & $\begin{array}{l}\text { counterion/nanoparticle } \\
\text { interactions }\end{array}$ \\
\hline F-CFC & Central Force & Central Force \\
SRR-CFC & Stochastic Reflection & Central Force \\
F-SRR & Stochastic Reflection & Stochastic Reflection \\
\hline
\end{tabular}

actions only. In the second case, counterions interact with nanoparticles through the SRR scheme in addition to Coulomb interactions, and interactions between couterions only consist in Coulomb interactions (no WCA potential between counterions in this case). We call this method F-SRR, for Full Stochastic Reflection Rules. Within the F-SRR variant, the minimal distance of approach between a counterion and a nanoparticle is called $\sigma_{N P / c}=\left(\sigma_{N P}+\sigma_{c}\right) / 2$.

The main characteristics of the three methods are summarized in tab. I.

\section{E. Computation of the transport coefficients of solutes}

In order to understand the ability of the different MPC simulation techniques to be quantitatively predictive in terms of transport coefficients, we compute the self-diffusion coefficient and the electrical conductivity of the solution. These quantities can be obtained from the computation of the integrals over time of adequate correlation functions.

The self-diffusion coefficient of solutes is computed from the autocorrelation function of the velocity:

$$
D=\lim _{t \rightarrow \infty} \frac{1}{3} \int_{0}^{t} \mathrm{~d} t^{\prime}\left\langle\left(\mathbf{v}_{i}\left(t_{0}\right) \cdot \mathbf{v}_{i}\left(t^{\prime}+t_{0}\right)\right\rangle_{t_{0}}\right.
$$

or using the mean square displacements:

$$
D=\lim _{t \rightarrow \infty} \frac{\left\langle\left(\mathbf{r}_{i}\left(t_{0}+t\right)-\mathbf{r}_{i}\left(t_{0}\right)\right)^{2}\right\rangle_{t_{0}}}{6 t}
$$

We also define a time-dependent diffusion coefficient:

$$
D(t)=\frac{\left\langle\left(\mathbf{r}_{i}\left(t_{0}+t\right)-\mathbf{r}_{i}\left(t_{0}\right)\right)^{2}\right\rangle_{t_{0}}}{6 t}
$$

The electrical conductivity, which is a collective transport coefficient, is computed from the autocorrelation function of the electric current in the simulation box:

$\chi=\lim _{t \rightarrow \infty} \frac{e^{2}}{3 k_{B} T V} \int_{0}^{t}\left\langle\sum_{i=1}^{N} z_{i} \mathbf{v}_{i}\left(t_{0}+t^{\prime}\right) \sum_{i=1}^{N} z_{i} \mathbf{v}_{i}\left(t_{0}\right)\right\rangle_{t_{0}} d t^{\prime}$ 
where $k_{B}$ is the Boltzmann constant and $T$ the temperature, $V$ is the volume of the simulation box, $z_{i}$ the valency of ion $i, e$ the elementary charge and $N$ the total number of solute particles in the simulation box.

In what follows, these transport coefficients are divided by their value at infinite dilution, denoted by $D^{0}$ and $\chi^{0}$, where $\chi^{0}$ depends on $D^{0}$ through $\chi^{0}=\frac{\beta}{V} \sum_{i=1}^{N} z_{i}^{2} e^{2} D_{i}^{0}$.

\section{SYSTEMS UNDER STUDY AND PARAMETERS OF THE SIMULATIONS}

In order to examine the precision and the numerical efficiency of the three algorithms mentioned above, we study several systems which differ from their asymmetry in terms of size and charge. The types of systems for which the explicit description of counterions is important from a physical point of view and stays moderate from a computational point of view include solutions of proteins, small micelles, small inorganic particles such as polyoxometallates. With these systems in mind, we chose to constrain ourselves to the study of three systems.

The diameter $\sigma_{N P}$ of nanoparticles, which is involved in the direct interaction potential between them, and allows us to define the volume excluded to solvent particles and to counterions, takes two different values: Either $1 \mathrm{~nm}$ or $4 \mathrm{~nm}$. The nanoparticles are surrounded by neutralizing monovalent counterions with a diameter $\sigma_{c}$ equal to $0.35 \mathrm{~nm}$.

When the diameter of the nanoparticle is $1 \mathrm{~nm}$, its charge $Z_{N P}$ is either $-4 e$ or $-8 e$, with $e$ the elementary charge. These characteristics are close to those of a system used as a standard in electrokinetic measurements, especially in electroacoustic experiments ${ }^{47}$, namely the silicotungstate polyoxoanion $\left(\mathrm{SiW}_{12} \mathrm{O}_{40}\right)^{4-}$. We also investigate the case where the charge is equal to $-8 e$ instead of $-4 e$, because we expect a stronger electrostatic coupling with counterions, whose dynamic properties are difficult to account for in numerical simulations.

When the diameter of the nanoparticle is $4 \mathrm{~nm}$, its charge $Z_{N P}$ is equal to $-16 e$. The ratio $Z_{N P} / \sigma_{N P}$ takes thus exactly the same value as for the system with $\sigma_{N P}=1 \mathrm{~nm}$ and $Z_{N P}=-4 e$. These characteristics are here typical of aqueous micellar systems, such as DTABr, dodecyltrimethylammonium bromide, where micelles are spheres of radius $2 \mathrm{~nm}$ and charge $-16 e$, as measured by electric conductimetry ${ }^{29}$.

The three families of systems will be referred to in the following using the charge of nanoparticles: $Z_{N P}=-4 e$, $Z_{N P}=-8 e$, and $Z_{N P}=-16 e$. The concentration of the suspension is evaluated through the packing fraction of nanoparticles $\phi_{\mathrm{NP}}$, as it is usually done in electrokinetic theories of colloids. Theories of electrolytes usually focus on the electrolyte concentration, which here would be equal to the number of nanoparticles divided by the volume of the simulation box. It must be noticed that, at a given volume fraction $\phi_{\mathrm{NP}}$, the numerical concentration of nanoparticles with $Z_{N P}=-16 e$ is $4^{3}$ smaller than with $Z_{N P}=-4 e$ or $Z_{N P}=-8 e$. In section $\mathrm{IV}$, the volume fraction of nanoparticles is one percent $\left(\phi_{\mathrm{NP}}=0.01\right)$. In section $\mathrm{V}$, it varies between 0.005 and 0.04 .

For each system, the parameters used to simulate the MPC fluid bath are: $\alpha=130, \gamma=5, \lambda=0.1 a_{0}$, where we chose the size of the cubic collision cells $a_{0}$ as the unit length. The unit of mass is that of solvent particles, $m_{f}=1$, the unit of energy is $k_{B} T=1$, and the unit of time is $t_{0}=\sqrt{\frac{m_{f}}{k_{B} T}}=1$. The box length is the same in every case $\left(L_{b o x}=32 a_{0}\right)$, the volume fraction of solutes being varied by changing the number of solutes in the simulation box. The mass of nanoparticles is $M=150 m_{f}$ with $m_{f}$ the mass of solvent particles, and the mass of counterions is $m_{c}=10 m_{f}$. The repulsion parameter of the WCA interaction potential is the same in every case: $\epsilon_{\mathrm{WCA}}=1 k_{B} T . \sigma_{N P / f}$, which is the the size parameter of the nanoparticle/solvent WCA interaction potential in F-CFC and the radius of the nanoparticle in other schemes is equal to: $\sigma_{N P / f}=2.31 a_{0}$ in F-CFC and $\sigma_{N P / f}=1.81 a_{0}$ in F-SRR and SRR-CFC. The WCA size parameter for the nanoparticle/nanoparticle interaction is either $\sigma_{N P}=4.95 a_{0}$ in F-CFC or $\sigma_{N P}=3.88 a_{0}$ for the other schemes. The WCA size parameter $\sigma_{c}$ for the counterion/counterion interaction is 3 times smaller than $\sigma_{N P}$ for $Z_{N P}=-4 e$ and $Z_{N P}=-8 e . \sigma_{c}$ is 12 times smaller than $\sigma_{N P}$ for $Z_{N P}=-16 e$.

The computed diffusion coefficients of solutes are in principle affected by the finite size of the simulation box. It was shown previously (see e.g. ${ }^{21}$ ) that for $L_{b o x}=32 a_{0}$ and the parameters we use here, this finite size effect is negligible. As for the nanoparticles, the diffusion coefficient at infinite dilution $D^{0}$ was extrapolated at zero volume fraction from the values of the diffusion coefficients $D$ of neutral solutes at low concentration. We have obtained $D^{0}=0.0109 a_{0}^{2} / t_{0}$ in every case. As for the counterions, we have taken the value of the diffusion coefficient at infinite dilution computed in our previous paper $^{36}$ in the same conditions as in the present work: $D^{0}=0.0418 a_{0}^{2} / t_{0}$. The results presented in following sections were obtained by averaging over 7 independent trajectories of duration between $5 \cdot 10^{5} t_{0}$ (F-CFC and SRR-CFC schemes) to $10^{7} t_{0}$ (F-SRR scheme).

\section{COMPARISON OF THE DIFFERENT SIMULATION SCHEMES}

\section{A. Numerical efficiency}

When the F-CFC algorithm is used, the time step of the simulation is $\Delta t=0.01 t_{0}$ and the time between two collision steps is $\delta t_{c}=0.1 t_{0}$. In the SRR-CFC scheme, SRR rules for solvent particles in the vicinity of nanoparticles are used instead of pair interaction forces. The time step is the same as in the F-CFC scheme: It can't be increased because of the combination of short-range 
repulsions and strong attractive electrostatic interactions between counterions and nanoparticles. If $\Delta t$ is too large, the discretized equation of motion of counterions deviates too much from the exact trajectory. Nevertheless, simulations with the SRR-CFC scheme are more than two times faster than with the F-CFC scheme, thanks to the SRR rules for solvent particles. The F-SRR algorithm combines two main advantages. First, the use of the SRR rules for solvent particles is more efficient than direct interactions, and second, the time step $\Delta t$ can be increased by a factor 10 . This is possible because the integration of the equations of motions of counterions does not involve a pair interaction potential combining a short-range repulsion and a long-range attraction anymore. We have then with the F-SRR scheme: $\Delta t=0.1 t_{0}$ and $\delta t_{c}=1 t_{0}$. Finally, simulations with the F-SRR scheme are found to be almost twenty times faster than with the F-CFC scheme for the systems investigated here.

\section{B. Radial distribution functions}

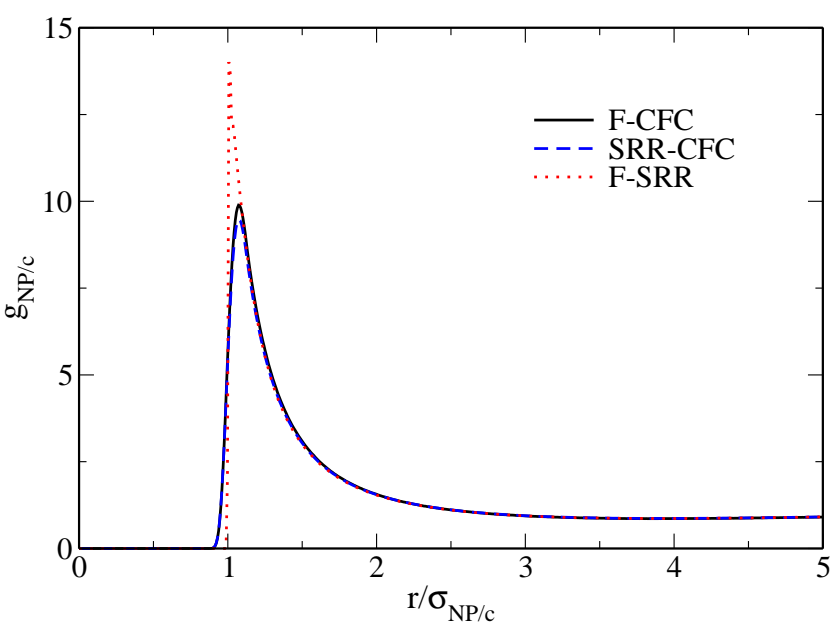

FIG. 1: Radial distribution functions between nanoparticles and counterions for the system $Z_{N P}=-16 e$ at the volume fraction $\phi_{\mathrm{NP}}=0.01$ obtained with the SRR algorithm for counterions (dotted red, F-SRR algorithm), a WCA interaction potential between counterions and nanoparticles and SRR for the solvent (dashed blue, SRR-CFC algorithm), and full WCA interactions (plain black, F-CFC algorithm). $\sigma_{N P / c}$ stands for the sum of counterions and nanoparticles radii: $\sigma_{N P / c}=\left(\sigma_{N P}+\sigma_{c}\right) / 2$.

To validate the new scheme, where SRR is applied for counterions in the vicinity of nanoparticles (F-SRR), the first thing to check is that the spatial organization of the solution is well reproduced. In Fig. 1, we give the radial distribution functions (rdfs) between counterions and nanoparticles obtained with the three different algo-

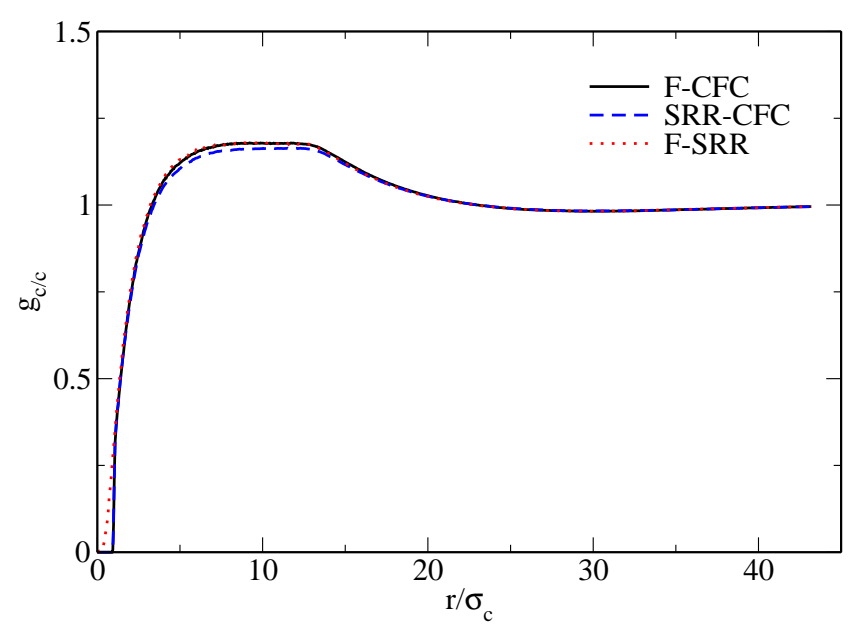

FIG. 2: Radial distribution functions between counterions for system $Z_{N P}=-16 e$ at the volume fraction $\phi_{\mathrm{NP}}=0.01 \mathrm{ob}-$ tained with the SRR algorithm for counterions (dotted red, FSRR algorithm), a WCA interaction potential between counterions and nanoparticles and SRR for the solvent (dashed blue, SRR-CFC algorithm), and full WCA interactions (plain black, F-CFC algorithm). $\sigma_{c}$ stands for the WCA diameter of counterions.

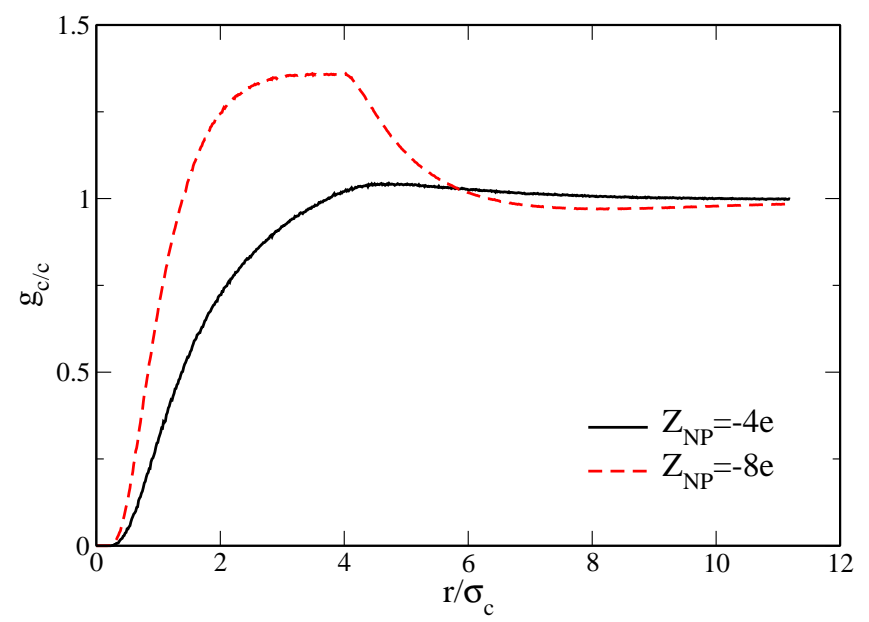

FIG. 3: Radial distribution functions between counterions for systems $Z_{N P}=-4 e$ (plain black) and $Z_{N P}=-8 e$ (dashed red) at a volume fraction $\phi_{\mathrm{NP}}=0.01$ obtained with the $\mathrm{F}$ SRR algorithm.

rithms, F-SRR, SRR-CFC and F-CFC. We obtain an excellent agreement between these methods. As expected, the rdf does not depend on the solvent/nanoparticle interaction (agreement between SRR-CFC and F-CFC). A small difference at contact appears between results 
obtained with the F-SRR scheme and other ones, because the interaction between counterions and nanoparticles is an effective hard-core interaction when the FSRR scheme is used. Even if the WCA interaction potential is strongly repulsive at short range, it is not an hard-core potential. The agreement is also excellent for the rdf between counterions displayed in Fig. 2. The good prediction of structural properties by the F-SRR schemes is an argument to show that counterions are adequately thermostated by the stochastic reflections. It also suggests that the structure of the cloud of counterions around nanoparticles is not affected by artefacts of the simulation procedure, such as multiple reflections. We double-checked this by computing the temperature using only the velocities of counterions, which confirmed their perfect thermostating.

Moreover, the study of rdfs allows us to check that the chosen systems are representative of qualitatively different regimes in terms of electrostatic coupling. (i) Systems $Z_{N P}=-4 e$ and $Z_{N P}=-16 e$ show no clear sign of electrostatic condensation, which was checked by computing the coordination numbers for counterions as function of the distance, $N_{c}(r)$. There is no clear inflexion point of the curve $N_{c}(r)$, which would be a signature of electrostatic condensation (Bjerrum criterion ${ }^{10}$ ). The counterion/counterion rdfs also show that interionic correlations within the electrostatic double layer are relatively weak (see Figs. 2 and 3). (ii) Conversely, the system $Z_{N P}=-8 e$ is a good model for highly charged nanoparticles moving with electrostatically condensed counterions. First, as expected, at a given volume fraction, the peak of the rdf between counterions and nanoparticles is higher for system $Z_{N P}=-4 e$ than for system $Z_{N P}=-8 e$, where the electrostatic interaction increases (see Fig. 4.). Second, the correlation between counterions is stronger in the system $Z_{N P}=-8 e$ than for $Z_{N P}=-4 e$, as it can be seen in Fig. 3. Third, the counterion coordination number $N_{c}(r)$ (not shown here) displays an inflexion point as a function of distance, typical of ionic condensation.

Finally, as the correlation between counterions increases from $Z_{N P}=-4 e$ to $Z_{N P}=-16 e$ and then to $Z_{N P}=-8 e$ (see Figs. 2 and 3 ), the electrostatic coupling between the nanoparticles and their counterions increases in the same way. The kink in the rdfs between counterions which appears at $r / \sigma_{c}=13$ for $Z_{N P}=-16 e$ (Fig. 2 ) and $r / \sigma_{c}=4$ for $Z_{N P}=-4 e$ or $Z_{N P}=-8 e$ (Fig. 3) corresponds to the maximal distance between two counterions situated in the vicinity of a given nanoparticle. This maximal distance is reached when two counterions are diametrically opposed to each other on a nanoparticle, i.e. at a distance equal to $\left(\sigma_{N P}+\sigma_{c}\right) / \sigma_{c}$. For $Z_{N P}=-16 e$, the WCA size parameter of counterions $\sigma_{c}$ is 12 times smaller than that of nanoparticles, so that the kink appears at about $r / \sigma_{c}=13$; For $Z_{N P}=-4 e$ or $Z_{N P}=-8 e$, the diameter of counterions $\sigma_{c}$ is about 3 times smaller than that of nanoparticles, so that the kink appears at about $r / \sigma_{c}=4$.

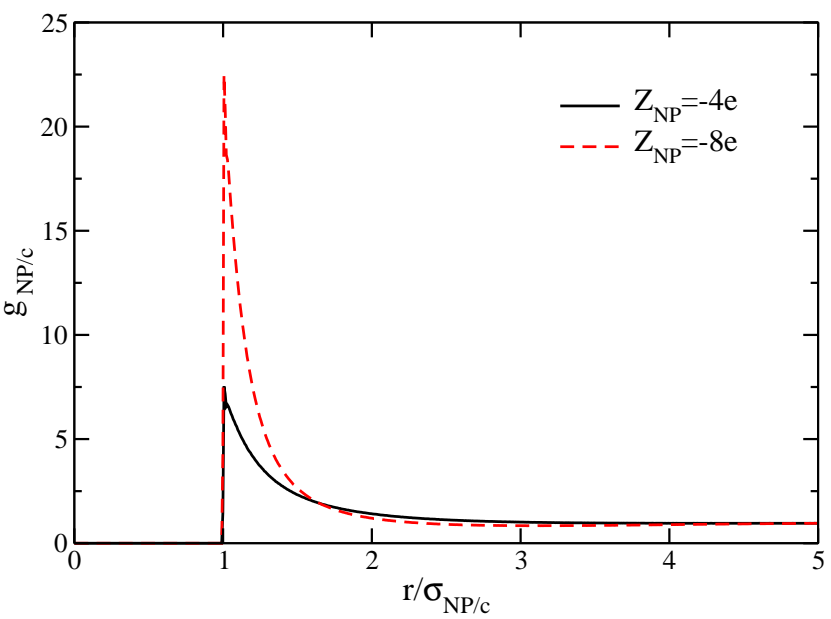

FIG. 4: Radial distribution functions between counterions and nanoparticles for systems $Z_{N P}=-4 e$ (plain black) and $Z_{N P}=-8 e$ (dashed red)at a volume fraction $\phi_{\mathrm{NP}}=0.01$, obtained with the F-SRR algorithm.

\section{Dynamic properties of solutes}

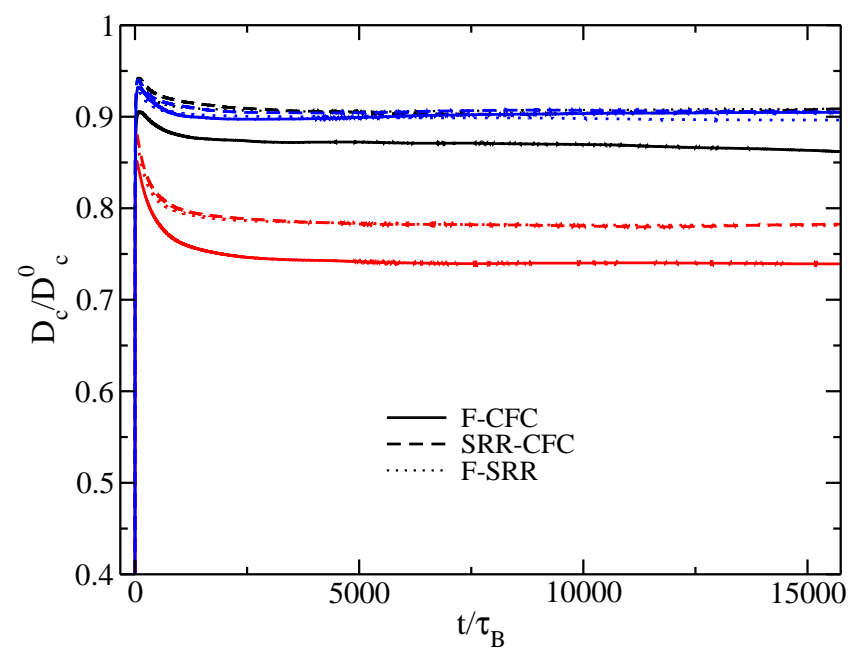

FIG. 5: Diffusion coefficient of counterions as a function of time for the three different systems (black: $Z_{N P}=-4$, red: $Z_{N P}=-8$ and blue: $\left.Z_{N P}=-16\right)$ obtained with the F-CFC (plain line), SRR-CFC (dashed line) and F-SRR (dotted line) algorithms at the volume fraction $\phi_{\mathrm{NP}}=0.01$.

We present in Figs. 5 and 6 the diffusion coefficients of nanoparticles and of counterions respectively, as functions of time. The time is rescaled by $\tau_{B}$ which is the Brownian relaxation time, i.e. the characteristic time 


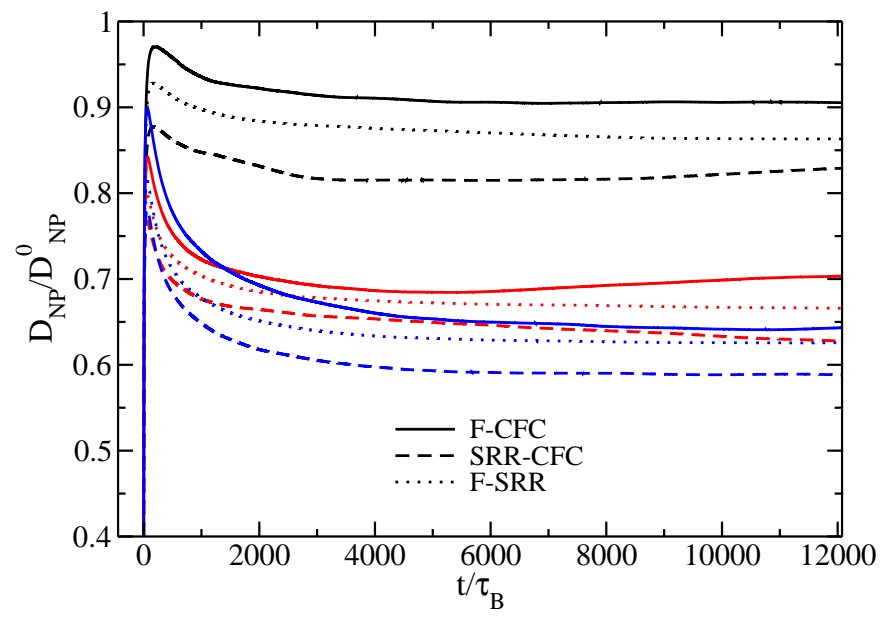

FIG. 6: Diffusion coefficient of nanoparticles as a function of time for the three different systems (black: $Z_{N P}=-4$, red: $Z_{N P}=-8$ and blue: $\left.Z_{N P}=-16\right)$ obtained with the F-CFC (plain line), SRR-CFC (dashed line) and F-SRR (dotted line) algorithms at the volume fraction $\phi_{\mathrm{NP}}=0.01$.

over which the velocity correlations of nanoparticles decay. These quantities were deduced from the mean square displacements divided by time. They are presented for the three algorithms, F-CFC, SRR-CFC, and F-SRR, and for the three systems $Z_{N P}=-4 e, Z_{N P}=-8 e$ and $Z_{N P}=-16 e$, for a unique volume fraction $\left(\phi_{\mathrm{NP}}=0.01\right)$. The diffusion coefficients are divided by their value at infinite dilution extrapolated from simulations with only a few solutes in the simulation box.

In every case, the diffusion coefficient is smaller than its value at infinite dilution. This is expected from electrolyte transport theories ${ }^{39,48}$ and was also obtained with other simulation techniques, such as Brownian Dynam$\operatorname{ics}^{16,49,50}$. In the framework of usual theories of electrolytes, the decrease of the diffusion coefficient as a function of concentration is assumed to be mainly due the electrostatic coupling between a solute and its ionic atmosphere, accounted for by the so-called electrostatic relaxation force. Hydrodynamic interactions also have an influence on this quantity as was shown for example by BD simulations ${ }^{29,51-53}$ : They increase the diffusion coefficient compared to situations where they are neglected, because hydrodynamic couplings tend to decrease the amplitude of the electrostatic relaxation.

As for counterions (Fig. 5), the decrease of the diffusion coefficient due to the combined effect of electrostatic interactions and hydrodynamics is moderate for systems $Z_{N P}=-4 e$ and $Z_{N P}=-16 e$, with a decrease of about 10 percent whatever the method. The decrease is significantly more pronounced for the system $Z_{N P}=-8 e$ : This is a dynamical signature of the electrostatic condensation of counterions on nanoparticles in this case. As condensed counterions move with the larger and slower nanoparticle, they diffuse slower than free ions: In average, counterions are slowed down.

The diffusion coefficients of counterions obtained by F-SSR and SRR-CFC are in excellent agreement. Both schemes only differ in the treatment of the dynamics of counterions in the vicinity of nanoparticles: Explicit WCA interactions in the SRR-CFC algorithm, and effective hard core interactions in the F-SRR one. The difference between results obtained by these methods is below the statistical noise of our calculations. First of all, this means that the simplified description of the dynamics of counterions in the vicinity of nanoparticles in the F-SRR scheme is adequate. Moreover, this suggests two things: (i) the small difference in the structure of the ionic clouds observed in the rdfs in Fig. 1 does not influence the dynamics of counterions; (ii) as long as counterions cannot penetrate the core of nanoparticles, the friction induced by the nanoparticle/counterion coupling does not depend on the mechanism by which counterions are excluded from the nanoparticles. Also, the CC coupling between counterions and solvent tends to equalize the velocities of ions and solvent molecules so that solvent velocities impose the counterion ones at the surface of the nanoparticle. The solvent evolves through the SRR scheme, which mimics no-slip boundary conditions: They are transmitted to counterions in the SRR-CFC scheme.

The comparison of F-CFC and SRR-CFC schemes is particularly interesting for counterions. These methods differ from the treatment of the nanoparticle/solvent coupling but do not differ from the treatment of the nanoparticle/counterion coupling. The results show a quantitative effect of the solvent-nanoparticle boundary condition on the friction felt by counterions for both systems $Z_{N P}=-4 e$, and $Z_{N P}=-8 e$. We remind the reader that the solvent-counterion coupling (within the collision steps) does not change in the methodologies we are comparing here. We interpret this effect as follows: In the full CFC algorithm (F-CFC), the solvent-nanoparticle interactions result in effective slip boundary conditions, which means that the trajectories of the solvent particles are less coupled to those of the nanoparticles than in the case of the SRR algorithm (the latter models no-slip boundary conditions). This differenceis transmitted to counterions through the solvent. As a result, the hydrodynamic coupling between counterions and nanoparticles is smaller within the F-CFC methodology than within the SRR-CFC one. As hydrodynamic effects reduce the electrostatic couplings, the electrostatic friction is more important with the F-CFC scheme. In other words, the stronger is the hydrodynamic coupling, the weaker is the electrostatic friction; The less intense is the friction, the higher is the diffusion coefficient. For the system $Z_{N P}=-8 e$ for instance, this explanation may account for the increase of the diffusion coefficient from about $0.73 D^{\circ}$ with F-CFC to $0.78 D^{\circ}$ with SRR-CFC.

The picture is slightly less straightforward for the transport of nanoparticles (Fig. 6). For all three sys- 
tems, there are differences for the computed values of the diffusion coefficient $D$ using the three methodologies, with the same sequence $D(\mathrm{~F}-\mathrm{CFC})>D(\mathrm{~F}-\mathrm{SRR})$ $>D$ (SRR-CFC). The values obtained from the F-SRR and SRR-CFC methods do not exactly agree for a given system, contrarily to what was observed for counterions, but the difference between the diffusion coefficients stays relatively small, around $0.02 D^{\circ}$, i.e close to the limit of statistical significance (the number of nanoparticles in the simulation box is smaller than that of counterions, which decreases the precision of the evaluation of $D$ ). Moreover, the ratio of diffusion coefficients of the different systems is almost the same for all three methods: $D\left(\mathrm{Z}_{N P}=-4 \mathrm{e}\right) / D\left(\mathrm{Z}_{N P}=-16 \mathrm{e}\right)=0.713$ for $\mathrm{F}-\mathrm{CFC} ; 0.725$ for F-SRR and 0.715 for SRR-CFC. This comparaison of relative transport coefficients makes sense in an analytical perspective, as experimental transport quantities are often obtained through the use of calibration curves using referent materials.

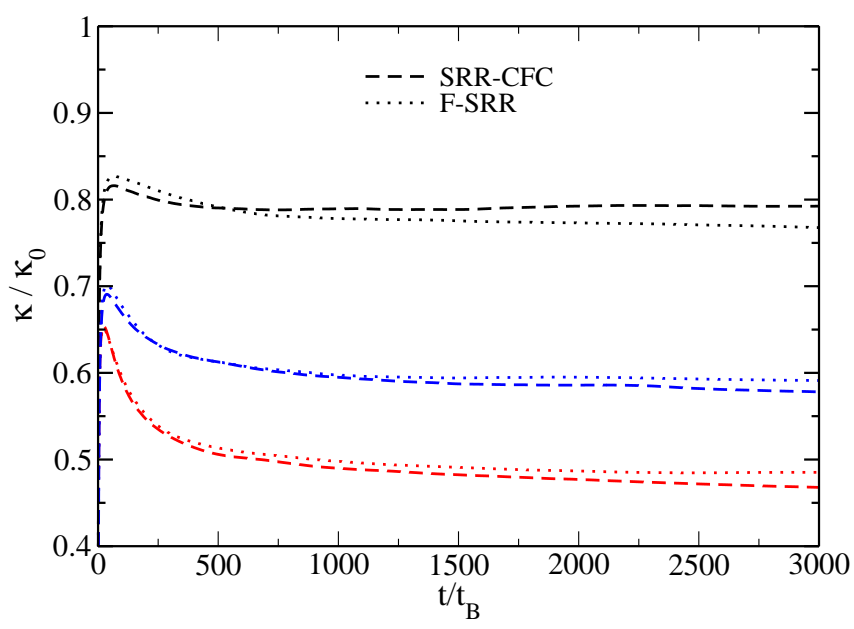

FIG. 7: Electrical conductivity of the suspension for the three different systems (black: $Z_{N P}=-4$, red: $Z_{N P}=-8$ and blue: $\left.Z_{N P}=-16\right)$ obtained with the SRR-CFC (dashed line) and F-SRR (dotted line) algorithms at the volume fraction $\phi_{\mathrm{NP}}=0.01$.

Collective transport coefficients are more difficult to compute than individual transport coefficients, as they require longer trajectories, but they can give complementary information. In particular, the electrical conductivity is particularly sensitive to the coupling between the nanoparticles and their counterions, since under an electric field, these two types of particles move in opposite direction relative to the electric field. Hydrodynamics couplings strongly reduce the ability of particles to move with opposing velocities, and thus considerably decrease the conductivity of the fluid. The integral of the autocorrelation function of the electric current divided by the electrical conductivity at infinite dilution is shown as a function of time in Fig. 7 for all three systems, and for the two counterion/nanoparticle coupling schemes (SRRCFC and F-SRR). The long time limit of this integral is the electric conductivity of the fluid. The agreement between both methods is again very satisfying.

All together, the comparisons between SRR-CFC and F-SRR methods make a strong argument for the use of the Stochastic Reflection Rules for counterions. It yields computed dynamical quantities which are very close to those obtained with the use of a central repulsive force, and it enables to avoid instabilities due to the discretization of the trajectories within rapidly varying energy landscapes. Finally, as already stated before, the F-SRR scheme is more efficient from the computational point of view.

\section{APPLICATION: INFLUENCE OF THE VOLUME FRACTION ON THE DYNAMIC PROPERTIES}

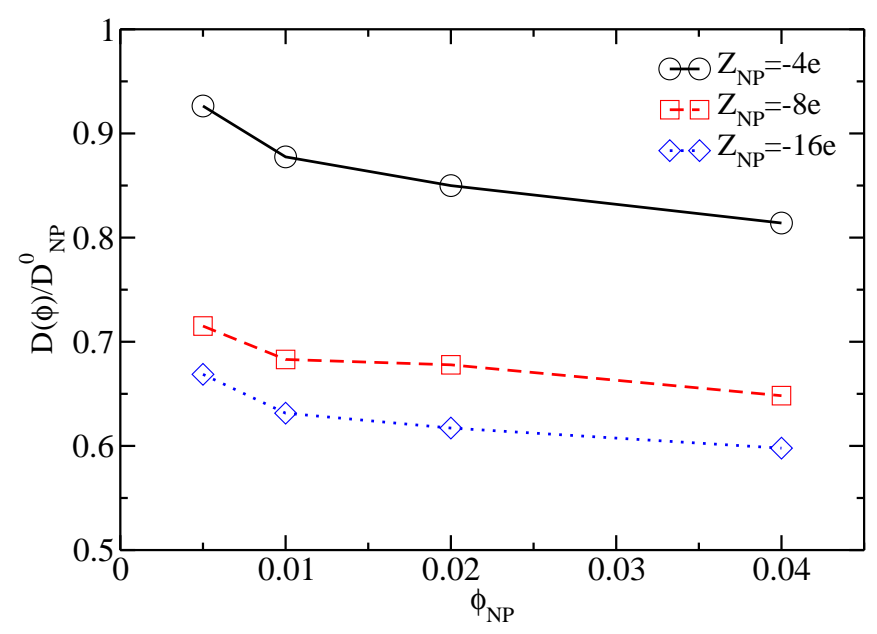

FIG. 8: Diffusion coefficient of the nanoparticles computed with the F-SRR algorithm as a function of the volume fraction of the solutes, for the three different systems (plain black: $Z_{N P}=-4$, dashed red: $Z_{N P}=-8$ and dotted blue: $Z_{N P}=$ $-16)$.

As we showed previously, the F-SRR proposed methodology is able to predict reliable transport coefficients for suspensions of nanoparticles and their counterions in the framework of multiparticle collision dynamics, and is faster than alternative MPC coupling schemes. We present hereafter (Figs. 8-10) series of results obtained within this methodology as a function of the volume fraction of nanoparticles $\phi_{N P}$.

The diffusion coefficients of nanoparticles shown in Fig. 8 decrease monotically with the volume fraction of the solute, as expected from an increase of the effect of re- 


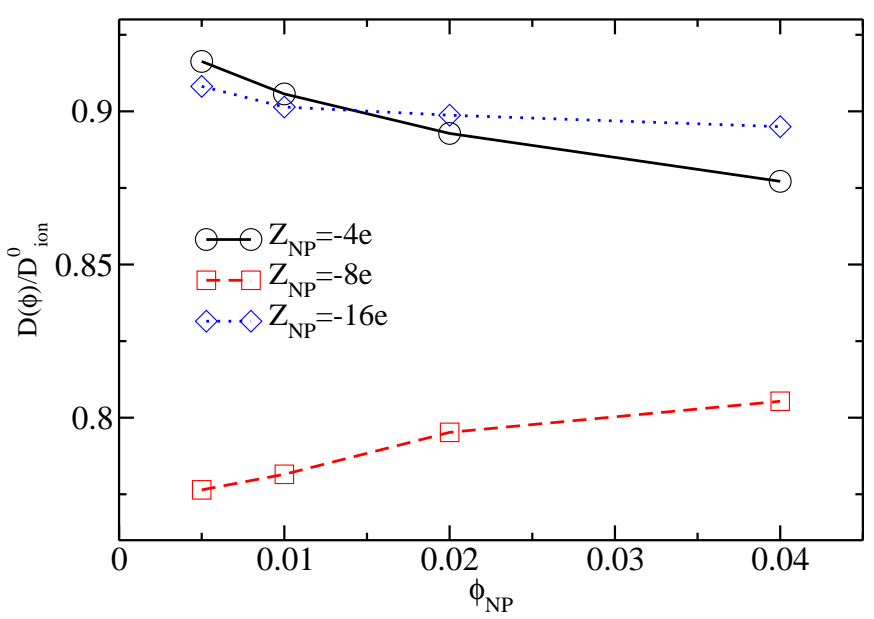

FIG. 9: Diffusion coefficient of the counterions computed with the F-SRR algorithm as a function of the volume fraction of the solutes, for the three different systems (plain black: $Z_{N P}=-4$, dashed red: $Z_{N P}=-8$ and dotted blue: $Z_{N P}=$ $-16)$.

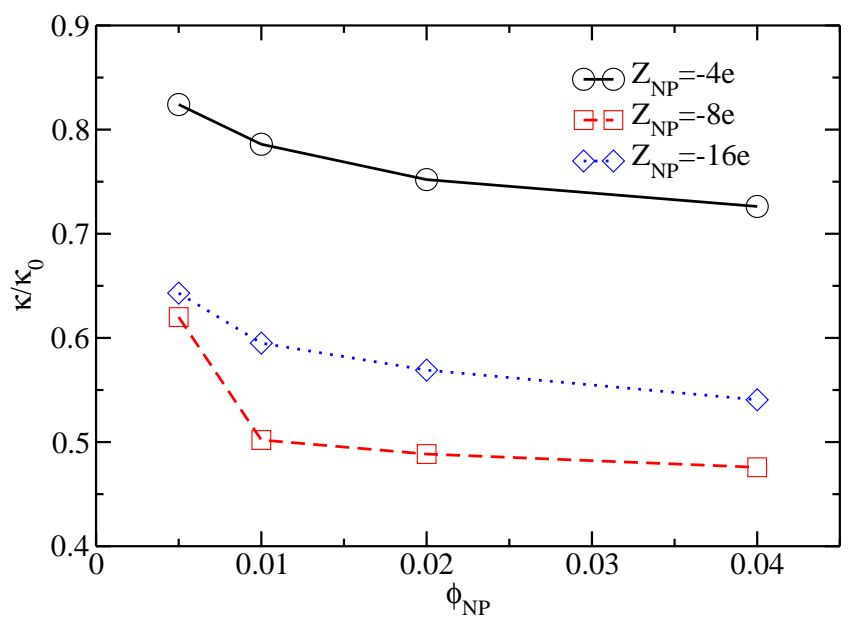

FIG. 10: Electrical conductivity of the suspension computed with the F-SRR algorithm as a function of the volume fraction of the solutes, for the three different systems (plain black: $Z_{N P}=-4$, dashed red: $Z_{N P}=-8$ and dotted blue: $Z_{N P}=$ $-16)$.

pulsive interactions that slow down the overall dynamics. Indeed, the free space available for the diffusion of the particles decreases when the density increases. For a given volume fraction, the fastest nanoparticles are the smallest and less charged ones (system $Z_{N P}=-4 e$ ). The diffusion coefficient of the most charged nanoparti- cles $\left(Z_{N P}=-16 e\right)$ is the smallest one, even if the ratio $Z_{N P} / \sigma_{N P}$ is the same for this system and for that with $Z_{N P}=-4 e$. This is an effect of the electrostatic friction of counterions: The attraction between counterions and nanoparticles is almost the same for $Z_{N P}=-4 e$ and $Z_{N P}=-16 e$ but the number of counterions surrounding the nanoparticles is much larger.

As for counterions, the results displayed on Figure 9 reveal two regimes: (i) for the less electrostatically coupled systems $\left(Z_{N P}=-4 e, Z_{N P}=-16 e\right)$ the diffusion coefficients of the counterions show a monotic decrease with the density in solutes; (ii) oppositely, for the systems containing electrostatically condensed ions $\left(Z_{N P}=-8 e\right)$, the diffusion coefficients of the counterions have a monotonic increase with the density in solutes. This counterintuitive result is due to an electrostatic effect: The ionic condensation of counterions on nanoparticles decreases when the global concentration of the system increases, because the electrostatic screening increases. Counterions are thus less attracted to nanoparticles and can diffuse faster. This behavior has already been observed in related systems by using Brownian Dynamics simulations $^{54,55}$.

The electrical conductivity of the fluid displayed in Fig. 10 decreases monotically with the density in solutes. For the systems $Z_{N P}=-8 e$, the fact that the diffusion coefficient of couterions increases with increasing concentration does not yield an increase of the conductivity with increasing concentration. It is noteworthy that the deviation from ideality (case without any interactions between solutes) is very high for systems $Z_{N P}=-8 e$ and $Z_{N P}=-16 e$. The analytical theories of transport in electrolytes often rely on the calculation of corrections relative to the ideal case ${ }^{39}$. By principle, they cannot be applied for such strong deviations from ideality.

\section{CONCLUSION}

In this study, we applied the MPC simulation technique to study suspensions of highly asymmetric electrolytes, i.e. charged nanoparticles and their counterions in a solvent. While the asymmetry between nanoparticles and counterions is in principle a source of computational difficulty, we took advantage of it, and we chose to couple the dynamics of these two kinds of solutes to the MPC solvent with a rule adapted to their respective size.

Counterions were coupled to the MPC solvent bath during the collision step. This results in an effective hydrodynamic size of counterions which is different from their real size, but the coupling between the solvent bath and solutes is simple, computationally efficient, and rather pertinent for small ions whose hydrodynamic radius is not unambiguously defined. Nanoparticles were coupled to the solvent particles either through a direct interaction force or with stochastic rotation rules which mimic stick boundary conditions.

Difficulties arising from the coupling between the MPC 
solvent and solutes have already been addressed by other studies, but our work is the first one which combines two different coupling schemes for two different types of solutes in the same simulation. This mixed coupling rule seems particularly adapted for suspensions containing solutes with different sizes. We studied systems with a size ratio between solutes from 3 to 12 , and this mixed coupling rule can be used in principle in MPC simulations devoted to suspensions of solutes of different sizes, within size ratios of the same order as in our study.

Moreover, we adapted the simulation procedure to address a second issue: The treatment of the strong electrostatic interactions in systems where both types of solutes have opposite charges, namely asymmetric electrolytes or nanoparticles and their counterions. We compared two different schemes to treat nanoparticles/counterions interactions. In both cases, the treatment of electrostatics at long range is similar, using the Ewald summation technique. But the short-ranged interaction potential, which is particularly difficult to sample as it combines a shortranged repulsion (counterions must be excluded from the volume of the nanoparticle) and a strong attraction (leading to an ionic condensation in some cases). In the first method, the short-ranged repulsion is modeled by a central repulsive force (WCA interaction potential). In the second method, counterions are reflected on the surface of nanoparticles. In other words, we described the dynamics of counterions in the vicinity of nanoparticles with two different schemes similar to the two usual nanoparticle/solvent coupling methodologies (central force coupling leading to slip boundary conditions or stochastic reflection rules leading to stick boundary conditions). We also investigated whether the nanoparticle/solvent coupling scheme had an influence on the dynamics of counterions.

The central result of our study is that the use of reflection rules for counterions at the surface of nanoparticles compares very well with the use of a central repulsive force. Moreover, the reflection rules are much more computationally efficient than the explicit computation of direct repulsive forces. This is an important result because the systems under study here belong to a domain of solute size which ranges between the electrolyte and the colloidal domains. Most analytical theories are expected to fail for such systems, and efficient simulation techniques are still missing. Thus, we did not intend here to confront our results to existing analytical or semi-analytical theories. Nevertheless, one disadvantage of the F-SRR method is that it is unable to represent detailed short-ranged interactions between the surface of the nanoparticle and the counterions.

In the last section, we also presented series of calculation of transport coefficients in suspensions of nanoparticles and their counterions for various densities. In every case the diffusion coefficient of nanoparticles is found to decrease with the volume fraction. As for the counterions, their diffusion coefficient is more affected when the electrostatic coupling with the nanoparticle increases, as expected. In one case, the diffusion coefficient of counterions is found to increase with the volume fraction of nanoparticles, a counterintuitive result which was already obtained in related systems by Brownian dynamics ${ }^{55}$. In the case of the electrical conductivity, the deviation from the ideal behavior (solutes at infinite dilution without any direct interactions) is strong: The conductivity is equal to half of its ideal value for the more electrostatically coupled system. For such systems, analytical electrolyte theories are meaningless, as they only make sense with small deviations from the ideal behavior ${ }^{39}$. Moreover, electrokinetic theories of colloidal systems, which usually relies on effective quantities such as the effective charge or the zeta potential, assume in most cases that colloidal particles do not contribute to the electrical conductivity $^{1,2}$. We remind the reader that the relative contribution of each species to the ideal electric conductivity scales as $n D z^{2}$, where $n$ is the density, $D$ is the diffusion coefficient and $z$ is the charge. For the system $Z_{N P}=-8 e$, the contribution to the electrical conductivity of nanoparticles is higher than the contribution of the counterions, which is therefore an indication that colloidal theories are not adapted either.

The present study is a first step towards a a more quantitative understanding of the dynamics of charged nanoparticles in solution. A complete comparison of MPC results to available analytical transport theories in the cases where analytical theories are reliable, and then novel extensions of electrolyte theories will be discussed in subsequent papers.

\section{ACKNOWLEDGMENTS}

Partial financial support of the Agence Nationale de la Recherche in the frame of the project Celadyct (ANR12-BS08-0017-01) is gratefully acknowledged. 
1 J. Lyklema, Fundamentals of Interface and Colloid Science, Elsevier: Amsterdam, 2005.

2 A. V. Delgado, F. Gonzalez-Caballero, R. J. Hunter, L. K. Koopal and J. Lyklema, Pure and Applied Chemistry, 2005, 77, 1753.

3 B. J. Berne and R. Pecora, Dynamic Light Scattering, Dover, 2000.

${ }^{4} \mathrm{H}$. Ohshima, Theory of Colloid and Interfacial Electric Phenomena, Academic Press, Amsterdam, 2006.

5 R. Pusset, S. Gourdin-Bertin, E. Dubois, J. Chevalet, G. Mériguet, O. Bernard, V. Dahirel, M. Jardat and D. Jacob, Phys. Chem. Chem. Phys., 2015, 17, 11779.

${ }^{6}$ V. Dahirel and M. Jardat, Curr. Opin. Colloid Interface Sci., 2010, 15, 2.

7 I. Lucas, S. Durand-Vidal, O. Bernard, V. Dahirel, E. Dubois, J. Dufrêche, S. Gourdin-Bertin, M. Jardat, G. Mériguet and G. Roger, Mol. Phys., 2014, 112, 1463.

${ }^{8}$ R. Kubo, J. Phys. Soc. Jap., 1957, 12, 570.

9 J. P. Hansen and I. R. McDonald, Theory of Simple Liquids, Elsevier: Amsterdam, 2006.

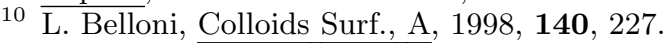

11 S. Marrink, H. Risselada, S. Yefimov, D. Tieleman and A. de Vries, JPC B, 2007, 111, 7812.

12 R. Groot and P. Warren, J. Chem. Phys., 1997, 107, 4423.

13 P. Turq, F. Lantelme and H. L. Friedman, J. Chem. Phys. 1977, 66, 3039

14 D. L. Ermak and J. A. McCammon, J. Chem. Phys., 1978, 69, 1352.

15 D. M. Heyes, Mol. Phys., 1996, 87, 287.

16 M. Jardat, O. Bernard, P. Turq and G. R. Kneller, J. Chem. Phys., 1999, 110, 7993.

17 P. J. Rossky, J. D. Doll and H. L. Friedman, J. Chem. Phys., 1978, 69, 4628.

18 D. M. Heyes and A. C. Brańka, Mol. Phys., 1998, 94, 447.

19 N. Bou-Rabee and E. Vanden-Eijnden, Commun. Pure Appl. Math., 2009, 63, 655.

20 A. Malevanets and R. Kapral, J. Chem. Phys., 1999, 110, 8605-8613.

21 J. Padding and A. Louis, Phys. Rev. E, 2006, 74, 031402.

${ }^{22}$ G. Gompper, T. Ihle, D. Kroll and R. G. Winkler, in Advanced Computer Simulation Approaches for Soft Matter Sciences III, ed. C. Holm and K. Kremer, Springer Berlin Heidelberg, 2009, vol. 221, pp. 1-87.

23 J.-P. Hansen and H. Löwen, Annu. Rev. Phys. Chem., 2000, 51, 209.

24 A. Laganapan, M. Mouas, A. Videcoq, M. Cerbelaud, M. Bienia, P. Bowen and R. Ferrando, J. Coll. Int. Science, $2015,458,241$

${ }^{25}$ V. Dahirel, M. Jardat, J.-F. Dufrêche and P. Turq, J. Chem. Phys., 2009, 131, 234105.

26 I. Pagonabarraga, B. Rotenberg and D. Frenkel, Phys. Chem. Chem. Phys., 2010, 12, 9566-9580.

27 B. Rotenberg and I. Pagonabarraga, Mol. Phys., 2013, 111, 827-842.

${ }^{28}$ M. Jardat, V. Dahirel, S. Durand-Vidal, I. Lucas, O. Bernard and P. Turq, Mol. Phys., 2006, 104, 3667.
${ }^{29}$ V. Dahirel, B. Ancian, M. Jardat, G. Mériguet, P. Turq and O. Lequin, Soft Matter, 2010, 6, 517.

30 J. D. Weeks, D. Chandler and H. C. Andersen, J. Chem. Phys., 1971, 54, 5237-5247.

31 J. T. Padding, A. Wysocki, H. Löwen and A. A. Louis, J. Phys.: Condens. Matter, 2005, 17, S3393.

32 J. K. Whitmer and E. Luijten, J. Phys.: Condens. Matter, 2010, 22, 104106.

33 A. Wysocki, C. P. Royall, R. G. Winkler, G. Gompper, H. Tanaka, A. van Blaaderen and H. Löwen, Faraday Discussions, 2010, 144, 245

34 I. O. Götze and G. Gompper, Phys. Rev. E, 2011, 84, 031404 .

35 J. Rotne and S. Prager, J. Chem. Phys., 1969, 50, 4831.

${ }^{36}$ G. Batôt, V. Dahirel, G. Mériguet, A. A. Louis and M. Jardat, Phys. Rev. E, 2013, 88, 043304.

37 M. Jardat, S. Durand-Vidal, P. Turq and G. Kneller, J. Mol. Liq., 2000, 85, $45-55$.

38 T. Yamaguchi, T. Akatsuka and S. Koda, J. Chem. Phys., 2011, 134, 244506.

39 J.-F. Dufrêche O. Bernard S. Durand-Vidal P. Turq, J. Phys.Chem. B, 2005, 109, 9873.

40 A. Botan, V. Marry, B. Rotenberg, P. Turq and B. Noetinger, J. Phys. Chem. C, 2013, 117, 978-985.

41 M. Ripoll, K. Mussawisade, R. G. Winkler and G. Gompper, Phys. Rev. E, 2005, 72, 016701.

42 S. Frank and R. G. Winkler, J. Chem. Phys., 2009, 131, 234905.

43 L. Jiang, N. Watari and R. G. Larson, Journal of Rheology, 2013, 57, 1177 .

44 C.-C. Huang, G. Gompper and R. G. Winkler, J. Chem. Phys., 2013, 138, 144902.

45 Y. Inoue, Y. Chen and H. Ohashi, Journal of Statistical Physics, 2002, 107, 85-100.

46 D. Frenkel and B. Smit, Understanding molecular simulation from algorithms to applications, Academic Press, 2002.

47 H. Hodne and J. K. Beattie, Langmuir, 2001, 17, 30443046 .

${ }^{48}$ L. Onsager and R. M. Fuoss, J. Phys. Chem., 1932, 36, 2689 .

${ }^{49}$ F. O. Raineri, M. D. Wood and H. L. Friedman, J. Chem. Phys., 1990, 92, 649.

50 M. D. Wood and H. L. Friedman, Zeit. Phys. Chem. N. F., 1987, 155, 121

51 M. Jardat, T. Cartailler and P. Turq, J. Chem. Phys., 2001, 115, 1066

52 M. Jardat, S. Durand-Vidal, N. da Mota and P. Turq, J. Chem. Phys., 2004, 120, 6268.

53 V. Dahirel, M. Jardat, J. F. Dufrêche and P. Turq, J. Chem. Phys., 2009, 131, 234105.

54 M. Jardat, B. Hribar-Lee and V. Vlachy, Soft Matter, 2012, 8, 954 .

${ }^{55}$ M. Jardat, B. Hribar-Lee, V. Dahirel and V. Vlachy, J. Chem. Phys., 2012, 137, 114507. 